\title{
Raumfahrtmedizin: Auf der Erde angekommen
}

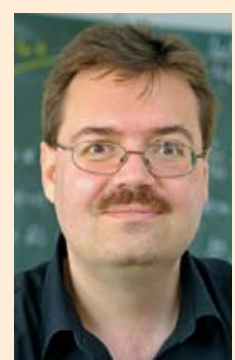

Deiver herich

Liebe Kolleginnen und Kollegen,

Forschen im Weltraum hilft uns in vielfacher Weise bei der Lösung von Fragen, die die Erde stellt. Viele wichtige Phänomene können überhaupt erst verstanden werden, wenn wir sie unter Weltraumbedingungen erforschen. Eine Trennung von sogenannter ,terrestrischer Forschung' und ,Forschung im Weltraum' ist schon lange nicht mehr zeitgemäß. Es geht immer um grundlegende wissenschaftliche Fragen, ob man sie nun besser auf der Erde oder besser im Weltraum untersucht. Oder beides miteinander verknüpft. Das geschieht in der weltweit einmaligen Forschungsanlage :envihab im Deutschen Zentrum für Luft- und Raumfahrt (DLR) in Köln (Seiten 180-185). Das :envihab ermöglicht die Erforschung des gesamten Menschen unter verschiedensten Umweltbedingungen. Hier ist die Raumfahrtmedizin - auch ganz praktisch betrachtet - ,auf der Erde angekommen'. Um medizinischen Nutzen aus Erkenntnissen von Weltraumexperimenten zu ziehen, müssen wir auch schon lange nicht mehr ins All: Neue Methoden zum Einsatz simulierter Schwerelosigkeit am Boden lässt auf die Herstellung komplexer Gewebe zum Einsatz bei Transplantationen hoffen (Seiten 173-178).

In der Raumfahrtmedizin bilden Parabelflüge das ,Rückgrat' der Forschung. Inzwischen gibt es auch Parabelflüge zu touristischen Zwecken, sodass der praktizierende Fliegerarzt daher vermehrt mit Personen konfrontiert sein wird, die eine medizinische Parabelflugtauglichkeit erlangen möchten (Seiten 166-171).

Vor gut 2 Jahren schrieb das DLR ein Stück Raumfahrtgeschichte: Am 1. November 2011 brachte die Trägerrakete „Langer Marsch $2 \mathrm{~F}^{\prime}$ das chinesische Raumschiff "Shenzhou-8“ vom Weltraumbahnhof Jiuquan in eine Erdumlaufbahn. An Bord: Die in Deutschland entwickelte und gebaute SIMBOX-Experimentapparatur mit insgesamt 17 deutschen und chinesischen Forschungsprojekten aus der Biomedizin (Seiten 200-204). Trotz gewaltiger logistischer und administrativer Herausforderungen, trotz verschiedener Kulturen, Denkweisen und unterschiedlichen Prozessabläufen, diese Mission wurde in Rekordzeit und mit großem Erfolg abgeschlossen. Denn Raumfahrtgeschichte haben hier auch alle die Menschen geschrieben, die durch Begeisterung, Mut und kontinuierliche, harte Arbeit das möglich gemacht haben. Wie auf kaum einem anderen Gebiet wird in der Raumfahrt sehr klar, zu was Menschen fähig sind, wenn sie gemeinsam an einem Ziel arbeiten.

Auch auf kaum einem anderen Gebiet müssen Fortschritte so hart erkämpft werden. Große internationale Teams mit den besten Wissenschaftlern arbeiten mit modernster Technologie daran, das Leben auf der Erde besser zu verstehen. Und tatsächlich ringen wir der Natur immer wieder und mit großem Aufwand ein Stückchen Erkenntnis ab. Tag für Tag arbeiten wir an kleinen Antworten auf große Fragen und Tag für Tag stoßen wir an unsere Grenzen. Und am Ende des Tages stehen wir dann immer noch wie staunende Kinder vor immer noch dem gleichen großen Wunder des Lebens, der Erde und des Weltalls. Das Wunder einer unvorstellbaren ordnenden Vernunft, die wir Naturgesetze nennen, und deren Teil der Mensch ist. Aber eine Erkenntnis ist uns beim Forschen unter Weltraumbedingungen ganz sicher: Diese Forschung zeigt uns wie keine andere unsere Grenzen auf, diese Forschung macht bescheiden.

Ihr

Prof. Dr. Dr. Oliver Ullrich, Zürich/Magdeburg 